\title{
Stimulating Intellectual Activity with Adaptive Environment (SMILE)
}

\author{
Marjan Gusev \\ University Ss Cyril and Methodius \\ Skopje, Macedonia \\ marjan.gushev@finki.ukim.mk
}

\author{
Short Paper
}

\author{
Jurij Tasic \\ University of Ljubljana \\ Ljubljana, Slovenia \\ jurij.tasic@gmail.com
}

\begin{abstract}
As of 2016, 47.5 million people have dementia worldwide according to the World Health Organization, and it is expected that this number will nearly double every 20 years, reaching 100 million sooner than 2030. The goal of the SMILE concept is to help the elderly to live independently and to prevent/delay dementia. It should improve the quality of life of elderly by introducing healthy habits and a lifelong involvement in mentally and socially stimulating activities. It should reduce social isolation, i.e. high degree of loneliness, dissatisfaction with social contacts, and decreased social network. The focus is on prevention of gradual decline in memory function and planning ability, as well. Applying social network concepts provides easier social interaction for older people. Using stimulating and interactive environment enhances and maintains brain plasticity through social interaction. So far there are no published articles or projects that are targeted at the same goal to develop a self-care social interactive TV system as a Service, which is the aim of the SMILE concept. There are a lot of projects and papers about the relevant technologies, however not the integrated approach, which is the key innovation in this concept.
\end{abstract}

\section{CCS CONCEPTS}

- Social and professional topics $\rightarrow$ Remote medicine; - Applied computing $\rightarrow$ Health care information systems; Health informatics; Interactive learning environments; • Hardware $\rightarrow$ Analysis and design of emerging devices and systems; $\bullet$ Software and its engineering $\rightarrow$ Cloud computing;

\section{KEYWORDS}

Adaptive environment, Social Networks, Tablets, Self-health Care, Avatars, elderly support, wearable telemedicine sensors

\section{ACM Reference format:}

Marjan Gusev, Shushma Patel, and Jurij Tasic. 2017. Stimulating Intellectual Activity with Adaptive Environment (SMILE). In Proceedings of BCI '17, Skopje, Macedonia, September 20-23, 2017, 4 pages.

https://doi.org/10.1145/3136273.3136283

Permission to make digital or hard copies of all or part of this work for personal or classroom use is granted without fee provided that copies are not made or distributed for profit or commercial advantage and that copies bear this notice and the full citation on the first page. Copyrights for components of this work owned by others than ACM must be honored. Abstracting with credit is permitted. To copy otherwise, or republish to post on servers or to redistribute to lists, requires prior specific permission and/or a fee. Request permissions from permissions@acm.org.

BCI '17, September 20-23, 2017, Skopje, Macedonia

(C) 2017 Association for Computing Machinery.

ACM ISBN 978-1-4503-5285-7/17/09..\$15.00

https://doi.org/10.1145/3136273.3136283

\section{INTRODUCTION}

Any deterioration in cognitive function, such as memory, thinking, behaviour and the ability to perform everyday activities is a syndrome of dementia. According to the World Health Organization [15] 47.5 million people have dementia and there are 7.7 million new cases every year. Although dementia mainly affects older people, it is not a normal part of ageing. It is one of the major causes of disability and dependency among older people worldwide and influences a strong physical, psychological, social and economic impact on caregivers, families, and society.

Worldwide, the costs of dementia will be even higher over time. There is a tentative estimate of an $85 \%$ increase in costs to 2030 , based only on predicted increases in the numbers of people with dementia [1]. The SMILE concept, as an interoperable ICT solution that uses open global standards to provide an adaptive environment, personalised and customised to each user contributes to the sustainability of health and care systems. The SMILE user scenarios based on user-led concepts aim at independent living of the elderly.

In order to provide a stimulating environment for older people, the SMILE concept sets focus on innovative models for service delivery, as well as on standardised and interoperable ICT platforms, services and data sources, making the solution modular and scalable. The SMILE concept is based on combining the traditional IPTV services with cutting-edge technologies regarding ambient control, social networks, cloud computing and personalised health monitoring.

To the best of our knowledge, there are no integrated commercial solutions, which can bring social network on IPTV, along with health self-care. The SMILE concept could make the ICT industry more competitive in the forthcoming years. Modularity and interoperability of the SMILE components, as well as, wide exploitation of each of the deployed technologies, guarantee scalability and a long-term influence of the integrated SMILE solution for the market. The technologies to be integrated by this concept are widely available, and accessible in a gender-neutral and accessible way, without ethical implications.

The paper is organised as follows. The SMILE concept and its objectives presented in Section 2. Section 3 presents the state-ofthe-art. Relevant issues are discussed in Section 4, and finally, conclusions and direction for further work in Section 5.

\section{SMILE CONCEPT AND OBJECTIVES}

The following objectives describe the SMILE concept's goal to preserve the vital intellectual shape of the service users, prolonging 
independence for longer: improved quality of life by mind stimulation with intellectual and entertainment content, social inclusion, personalised self-care system, a solution as a service.

A good Quality of Life from an individual's subjective perception means that their life is pleasant and valuable. Both external and internal components comprise Quality of Life. The former are established by the surrounding community (social services, transport, security etc), while the latter derived from ourselves: optimism, positive perception of health issues, the ability to adapt to given situations, and most importantly, a better attitude to face challenges and problems. Most commonly, the decline in senior citizens' Quality of Life is due to being unprepared for challenges such as retirement, family, society and health related issues that require adjustments to perceptions of their lives. Also, currently older people are passive consumers of media, experiencing no TV interactivity whatsoever.

By careful design of technology and the corresponding content it is possible to make the elderly take an active role and face life challenges more easily while minimising the decline of their own Quality of Life. Recent research (as elaborated further in this document) concludes that mind stimulation with appropriate interactive intellectual and entertainment content enhances the quality of life of the elderly. The important part of elderly health is mind health and willingness and joy in performing everyday activities.

User heart beat rate and other personalised health-related medical parameters would be monitored in real time by wearable sensors. All measured parameters are automatically updated in the self-care system that corresponds with online health related expert services and provides health-related advice. It is designed as a service ecosystem that connects the medical sensors and interactive TV with the cloud-based service enabling interactive self-care environment. Planned sensors include measurements of body temperature, blood pressure, body fat percentage, heart rate, ECG, blood glucose, etc.

The SMILE concept introduces mobile systems that recognise human activity in personal living environments. The systems autonomously integrate all available sensors around the user and self-configure to recognise user activities. A system that follows the users' daily activities helps the users to communicate with interactive IPTV by gestures or voice, and also by keyboard. The notification centre serves as a reminder for personalised therapies and activities the user should perform. The idea is based on the implementation of robust activity recognition systems, including cameras and microphones to enable voice-activated commands, presence detection and movement activation of commands. Subsequently, this enables smarter activity-aware energy management in buildings, and advanced activity-aware health assistants.

Integrating social networking and interactive TV (such as smart TVs or IPTV) has strong synergies with the area of health care; interactive activities and discussions; and consequently mind activities. However, it opens a very complex social space of new services achieving the best user acceptance. It indicates that social features in interactive TV services in general can be well accepted, even if they are quite immersive to the TV experience.

Future Internet and social networks need to include a number of Internet-connected sensors, including cameras, TV sets, speakers and microphones. Based on these sensors, emerging applications are able to collect, filter, analyse, process and store large amounts of data captured from the social networks, as well as related metadata captured as part of perceptive multimedia signal processing.

The ability to search this information in a scalable, effective, realtime and intelligent way can empower a wide range of added-value applications in the areas of surveillance, social networking, smart homes, security and more. In addition multimedia search engines for user profiled content should be able to support ambient/intelligent synthesis of related content in real-time.

The generic cloud-based ICT framework can support the whole design lifecycle, and the core architecture platform to host the SMILE solution needs to be based on virtualisation techniques to store and manage database, applications and cope with increased computing and communication demands. The dew computing scenario [3] includes a dew server that can be hosted in the smart living environment and take the responsibility to communicate with the cloud and perform a lot of micro services independently and autonomously.

Besides the autonomous function, the SMILE concept aims to integrate the social networking, so the communication to the cloud server is a necessity.

Current social computing networks target mainly the younger generations and are adapted to be used by personal computers, tablets and mobile phones. However, the human computer interface should be significantly changed in order to adapt it to older people, due to their resistance towards complex new features, and preference of using minimalistic design. The idea is to enable interactivity in a TV, not just by simple remote controllers, but simultaneously avoid complex mobile phone applications that are not convenient for the elderly with limited accessibility due to small size.

The overall concept is planned to use open source cloud computing technology solutions (e.g. OpenStack, Eucalyptus, Open Nebula, etc) and facilitates scalability and virtualisation, offering the possibility to support the Software as a Service (SaaS) model and dynamic service orchestration. In addition, containers are a promising technology instead of pure virtual stations, especially in the case of building a dew server. The idea is also to enable a platform without the need to install components but to enable service access by configuration through a web browser and Internet connection.

In addition, the cloud server needs to retain its performance regardless of the number of concurrent users, so the cloud needs to be carefully designed.

\section{STATE OF THE ART}

This section gives an overview of the projects towards elderly support.

There are several research results in eHealth area producing personalised medical sensors and their application in home appliances and assisted living devices. The latest research shows that even these solutions can be embedded in mobile phones and tablets realising the "As A Sensor" concept. These sensors can measure several essential parameters such as heart rate, ECG, body temperature, body pressure, oxygen saturation, body fat percentage, stress levels and even blood glucose. The new devices, attached to personal computers or embedded in mobile devices (such as mobile phones and 
tablets are known also as Independent Diagnostic Centres, such as LifeWatch SmartPhone V [9].

Mobile phones or smartphones are rapidly becoming the central personalised device. Application delivery channels are transforming mobile phones into App Phones with a growing set of cheap and powerful embedded sensors [8], and besides the standard ones (accelerometer, digital compass, gyroscope, GPS, microphone and camera) [7], the new revolutionary sensors include temperature, pressure, heart rate sensing. Mobile phone providers have even started to work in this area, for example, Android has developed a specialised platform and API how to approach and use the sensors, by acquiring raw sensor data, activation of sensor event listening, etc.

There are several research projects both in the EU and worldwide that target ambient intelligence applications, such as for example, MIMOSA [5] architecture and development platform that uses a microsystem technology to enable realisation of a low-cost and low power consumption, and small size system, which can also be used in the field of health care. The Spes project [11] aims at building interoperability standards and creating legal framework for such solutions.

There are a lot of ongoing and finished projects towards elderly support. For example, the Silver project [2] aims at developing a robotic assistance to support elderly living. The IN-LIFE project [6] offers all-around, personalised, multi-faceted existing ICT solutions and services addressing diverse daily activities (eating, physical activity, commuting, mental stimulation, communication, social interaction, etc.) to users with cognitive impairment living in their own home or in sheltered homes, as well as to their formal and informal carers.

Similarly, the Vital Mind project [14] aims at increasing the lifespan of the elderly by enabling them to actively participate in mind fitness exercises while watching TV. Not only does the SMILE concept allow the elderly to stay independent and active in their home environments, but also provides e-Health care by measuring blood pressure and blood sugar levels without the need to visit the hospital. The eWALL [13] is another EU funded project that aims at improving the elderly life. The eWall system architecture is composed of two main subsystems: eWALL Sensing Environment responsible for explicit and implicit interaction with the user and eWALL Cloud as central processing and data storage subsystem. Our system builds one step beyond with the integration of social networks, and is not based not just on the collection of various data about the user and its environment from medical and environmental sensors and control of environment through actuators, as the eWall system does.

The SMILE application integrates reminder, calendar, scheduler and alarm clock. Moreover, it serves as a companion and allows social networking. Additionally, medical devices for measuring blood pressure and blood glucose level may be connected in order to get tele-medical advice. In this way, all major needs of elderly people are being covered. The program is displayed on an interactive TV monitor with an embedded camera to detect movement. The software needs to be built as an interoperable environment and includes interfaces to different sophisticated devices for movement detection, such as XboX, etc.
The remote control can be a special remote device (without keyboard or numeric pad), or an application on an iPad or smartphone. The idea is to use a device with the following buttons only: on (like) / off (dislike) buttons, 4 arrow keys: up, down, left, right, an OK button in the centre, and 4 color-coded buttons: red, blue, green, yellow.

The avatar mainly communicates by sending audio messages to the user and displaying images. The sound channel is also used to accept commands or establish a communication to the system.

\section{DISCUSSION}

Demographic ageing is a worldwide process that shows the successes of improved health care over the last century. Many are now living longer and healthier lives and so the world population has a greater proportion of older people. Dementia mainly affects older people, although there is a growing awareness of cases that start before the age of 65 . By 2050, people aged 60 and over will account for $22 \%$ of the world's population.

Medical care in recent decades has significantly extended the life expectancy of people but also a probability of developing agerelated medical conditions. Dementia not only prevents people from living an independent life but also requires uninterrupted/permanent supervision. These conditions not only put an enormous burden on the relatives but also usually require institutionalised care.

Two essential aspects are directly targeted:

- mind intellectual stimulation, and

- enabling of self-health care sensors to be integrated in the system for providing essential medical care.

The add-on values are introduction of interactivity and integration of social media into a cloud-based solution.

By keeping the elderly in good intellectual shape as long as possible leads to the conclusion that an active population results in reduced medical assistance. It increases productivity of active population and at the same reduces time costs for social and medical services for the society as a whole.

The SMILE concept can improve independent living and social integration of the elderly and the disabled people by using interactive intellectual content and social communication scenarios. Furthermore, this concept encourages the elderly to strengthen their personal health care and allows them to live in their preferred home environment, thus reducing admissions and days spent in care institutions.

By entering the e-Health era, the SMILE concept reduces the time and money spending on visiting the doctors' and pharmacists' offices by providing the elderly with the opportunity to, for instance, measure heart rate, blood pressure and blood glucose levels at home. It can be realised by a set of personalised telemedicine sensors connected to or embedded in mobile devices, smartphones and tablets, or realised as add-on hardware (IoT) devices. By coupling this personalised health care system with an interactive TV solution and a social communication network, the outcomes of the implementation of this concept results in a healthier lifestyle of the elderly.

Society can benefit from seniors with a good quality of life after social and health service expenditures have been lowered by the aid of a personalised health-care system, which allows users to perform 
diagnostic and therapy counselling without having to leave their homes. The outcomes are intended only for those interested and capable of working with computers, tablets or devices that support interactive TV. All necessary equipment and gadgets are not cheap and there is a danger that only rich users will be able to afford the necessary equipment required to complete the whole infrastructure.

Involvement of Smart TV based personalised care model, or different available personal assistants, within the SMILE concept, can assist older people to live independently and will help the elderly to easily adapt to modern technology environments (e.g. introduction of new technology social media via a familiar media TV).

Since the SMILE concept establishes a network of virtual users, it also develops distant relationships without personal and physical contact. From the social and human point of view periodical opportunities for personal meetings, discussions and eye contacts are welcomed. It would give the whole concept an additional human value, which is usually neglected.

Regulation and social rules may dictate certain kinds of constraints. Security and individual privacy concerns may further complicate the discussions between participants; and also, the overall implementation of the system.

Several voice-activated systems were developed recently with different goals. Examples include iPhone Siri, Android Google Now Assistant or Google Now, Amazon Alexa or Echo dot etc. Most of them are made as a personal assistant and are developed primarily for mobile phone platforms, or are as an independent home box that can play music and interact with the user.

Our concept actually builds on an integration of recommender systems, personal voice-activated assistants, products towards elderly support and wearable telemedicine sensors. An example of integration challenges about ECG wearable sensors is given by Gusev et. al [4]. On the other side, the MIndGym approach [10, 12] is realised using EEG-based signals and recommendations about Mind cognitive skills of elderly with the goal to provide mind gymnastics to keep the mental health and elderly brain in a good shape. This is complimentary to the SMILE concept where the main goal is same but is based not on the scanned EEG and corresponding recommendations, but on providing an adaptive environment and stimulating intellectual activity.

The SMILE concept represents a wide range of system developments, methods of telemedicine, and social networking challenges. Its functionalities are fully deployed by the coexistence of remote telemedicine sensors and devices, interactive TV, personal assistants and cloud computing. Adaptive in this context means that it adapts to the user preferences. This concept builds on a methodology to design user-profiled contents is also one of the main outputs. This will be based on the directed feedback of the elderly, which will be further used to define business aspects on how to develop interoperable independent living solutions.

\section{CONCLUSION}

With the efficiency being based on developing simply designed and user-friendly interfaces, the proposed SMILE concept, and its implementation system are completely adaptable to older people's habits. The newly developed concept aims at empowering people with age-related dependencies to be able to live independently for longer. It also proposes social inclusion and mobility with seamless support in and outside the home.

The innovative approach combines different technologies, such as interactive TV, social networking, and Software as a Service (SaaS) cloud solutions in order to create a system for personalised healthcare and social inclusion and entertainment of the elderly, which enables them to stay healthy and active. Therefore, it impacts the life expectancy by keeping the elderly active and more independent for longer.

Achieving the coexistence of the different technologies requires multidisciplinary research, which consists of telemedicine-based health care, reinforcing achievement motivation in elderly people, multichannel social computing, cloud computing, etc. Most importantly, the system proposed herein is a specific platform with which governs the operational rules of all system functional components, and integration of different software and hardware components in a unified and intuitive environment.

In this paper, we have described the motivation to introduce such a system and its objectives. In more details we have elaborated the SMILE concept and approach to implementing an ICT-based system. The comparison to the ongoing projects and published research papers show that there is only partial overlapping in the integration of proposed technologies to achieve a proposed innovation. So far, the overall goal of the SMILE concept is not specified by any known project or published concept design. Future work is based on building a prototype and conducting experiments with the implemented SMILE concept.

\section{REFERENCES}

[1] Alzheimer's Disease International. 2016. World Alzheimer Report 2016: Improving healthcare for people living with dementia. (Sept 2016). https://www.alz.co. $\mathrm{uk} / \mathrm{research} /$ world-report-2016

[2] eWALL: EU FP7 project. 2016. Supporting Independent Living for the Elderly through Robotics. (2016). http://ewallproject.eu/

[3] Marjan Gusev. 2017. A Dew Computing Solution for IoT Streaming Devices. In Information and Communication Technology, Electronics and Microelectronics (MIPRO), 2017 40th International Convention on. IEEE, 415-420.

[4] Marjan Gusev, Aleksandar Stojmenski, and Ivan Chorbev. 2016. Challenges for development of an ECG m-Health solution. Journal of Emerging Research and Solutions in ICT 1, 2 (2016), 25-38.

[5] Civitas initiative. 2013. CIVITAS-MIMOSA, EU FP7 project. (2013). http://civitas. $\mathrm{eu} /$ content/mimosa

[6] INLIFE: H2020 project. 2016. INdependent LIving support Functions for the Elderly. (2016). http://www.inlife-project.eu/

[7] Wazir Zada Khan, Yang Xiang, Mohammed Y Aalsalem, and Quratulain Arshad. 2013. Mobile phone sensing systems: A survey. Communications Surveys \& Tutorials, IEEE 15, 1 (2013), 402-427.

[8] Nicholas D Lane, Emiliano Miluzzo, Hong Lu, Daniel Peebles, Tanzeem Choudhury, and Andrew T Campbell. 2010. A survey of mobile phone sensing. Communications Magazine, IEEE 48, 9 (2010), 140-150.

[9] LifeStar ACT Client Care Center. 2017. LifeWatch. (2017). www.lifewatch.com

[10] Shushma Patel, Dilip Patel, Marjan Gusev, Sasko Ristov, Jurij Tasic, and D RudanTasic. 2015. Mind gymnastics for good intellectual health of elderly peopleMindGym. In Cognitive Informatics \& Cognitive Computing (ICCI* CC), 2015 IEEE 14th International Conference on. IEEE, 309-316.

[11] Spes Project. 2014. (2014). http://www.spes-project.eu/

[12] Jurij Tasic, Darja Rudan Tasic, Andrej Vovk, Dusan Šuput, Shushma Patel, Dilip Patel, Marjan Gusev, and Sasko Ristov. 2015. Cognitive Processes of the Elderly Brain with MindGym Approach. International Journal of Software Science and Computational Intelligence (IFSSCI) 7, 4 (2015), 18-34.

[13] University of Toronto. 1995. eWALL - Home Caring Environment. (1995). http://sites.utoronto.ca/qol/projects/seniors.htm

[14] Vital Mind. 2011. (2011). http://www.vitalmind-project.eu/

[15] World Health Organization. 2016. Dementia fact sheet. (April 2016). http: //www.who.int/mediacentre/factsheets/fs362/en/ 